\title{
Physiotherapy and Foot Drop Corrections
}

\author{
W. M. LENNOX, F.R.G.s. (Eng.)
}

Leprosy neuritis affecting the lateral popliteal nerve is the commonest cause of foot drop in tropical countries. Tibialis posterior transfer has proved to be a very satisfactory operation for this condidion, and many of these operations are performed every year. Good results depend upon many factors, but one cause of failure is improper physiotherapy. Since the overall responsibility for cases selected for operation rests with the surgeon, it is intended in this paper to review some facets of physiotherapy for those who may wish to carry out tibialis posterior transfer, and who will be called upon to prescribe physiotherapy afterwards. Surgical technique will not be discussed, but may be reviewed from the references given at the end.

\section{Principles of Physiotherapy for Tendon Transfers}

(I) Pre-operatively - Isolation exercises for the muscle to be transferred.

(2) During plaster immobilization: General limb exercises, but not including movements in which the transferred muscle normally participates.

(3) After removal of plaster: (a) Unopposed contraction exercises.

(b) Opposed contraction.

(c) Protected function.

(d) Unprotected function.

The principles of physiotherapy in tendon transfer surgery are summarised in the table. In hand surgery, advantage can be taken of muscle synergism to simplify re-education, but after transfer, the basically pro-gravity tibialis posterior has to be educated to an antagonistic function. This is more difficult to achieve than is re-education of a muscle transfered from one function in a synergistic complex to another. However, with the tibialis anterior paralysed, voluntary inversion of the foot is due entirely to tibialis posterior, and this relative isolation is helpful to the therapist. Even so, re-education of this muscle requires close application on the part of all concerned, more so than when dealing with tendon transfers about the hand. As reeducation takes place in the brain, young and intelligent patients educate better and more quickly than the elderly and unintelligent.

The management is summarised in the figure. The aims of treatment are:

(a) to teach the patient to isolate the action of the muscle before operation;

(b) to exercise it in isolation after operation;

(c) to reintegrate it into the complex reflex pattern of walking
Pre-operative therapy: The tibialis posterior of the affected leg is exercised by placing the ankle across the opposite knee, and inverting against gravity. This exercise is repeated many times each hour until it can be performed without contraction of any other muscle in the calf. Initially, it is helpful for the physiotherapist to apply manual resistance to the foot, and to palpate the calf. It may take anything from one day to three weeks to isolate this muscle, and operation should not be performed until dissociation is complete. The exercise will not increase the strength of the muscle; any apparent enhancement of power represents increasing voluntary control. Patients who are slow to isolate may be helped by a small weight tied to the foot while they exercise.

During this period patients who have a tight tendoachilles undergo passive dorsiflexion stretching. Established contractures are never improved by this, but it sometimes enables borderline cases to avoid surgical lengthening.

Great stress should be placed upon the achievement of dissociation before operation. Time spent in isolation exercises before operation is an investment saving much time later.

Physiotherapy and Foot Drop Corrections 
Post-operative management: The leg remains in plaster for the time advised by the surgeon; five weeks is the safest minimum. For the first five or six days after operation the patient remains in bed with the limb elevated, and during this time straight leg raising and knee bending is practised. The weight of the cast forms a convenient load for the muscles, and the exercise maintains their tone, promotes circulation, encourages the absorption of exudates from the operation sites, and helps to minimise adhesions. After a wcek a walking rocker is added to the cast and the patient is ambulated and discharged from the ward with the injunction to continue the general exercises himself.

Patients who have difficulty in achieving dissociation pre-operatively can be helped to 'remember' by practising isolation exercises on the unoperated foot during their time in plaster.

Immediately after removal of the plaster the angle of the foot at rest, in maximum dorsiflexion, and in plantar flexion, is measured. These measurements are repeated at intervals as a record of progress.

The phase of post-operative isolation exercises now begins. The leg is crossed as before, and the patient attempts to invert, while watching his foot dorsiflex. The physiotherapist palpates the calf to detect contraction of other muscles. If other muscles are felt to contract, then the therapist must sit with the patient and help him 're learn' isolated contractions. After two or three days the patient will be accustomed to dorsiflex by attempting inversion, and the exercise may now be performed against gravity. Between exercise periods a plaster backslab is worn, the foot being heid at $75^{\circ}$ dorsiflexion, and it is most important that the patient should not at any time remove it except when exercising with his therapist. It is an advantage to have the patient in bed during this period, but failing this, he may non-weight bear on crutches.

Two points require special mention. A minority of patients on coming out of plaster, will be found to have forgotten how to contract their tibialis posterior in isolation. They exhibit a movement consisting of a plantar thrust (calf) followed by a recoil which mimics dorsiflexion. This pattern must be stopped before it becomes established. The physiotherapist must sit with the patient and carefully instruct and supervise until the correct movement can be performed. These patients require intensive personal attention, are slow to achieve dissociations, and may relapse if vigilance is relaxed.

The other point concerns the quality of muscle contraction. Many physiotherapists are content for their patients to practise 'Jerky' dorsiflexion contractions of short duration. It is desirable that once the movement of dorsiflexion is established that the muscle should perform sustained contractions in preparation for its function during walking. This type of contraction is also more effective in stretching adhesions. After the first few days, therefore, the patient is encouraged to carry out sustained contractions lasting two or three seconds, and of gradually increasing power. This culminates in the addition of weight to the foot, gradually increasing to about three or four hundred grammes.

Initially, exercise periods are kept short. On the first day two periods of not exceeding five minutes each should be allowed, increasing to three periods on the second day, and gradually lengthening thereafter. Each case must be managed on its merits. The appearance of warmth and/or swelling is an indication to slow down treatment or institute rest: if persistent, some complication (such as stitch sepsis) should be considered.

This initial phase of treatment ends when examination reveals complete dissociation of dorsiflexion from contraction of other calf muscles. Dissociation is decided by careful palpation of the calf during dorsiflexion, and by the following test. The patient dorsiflexes, and by means of a finger the examiner exerts gentle pressure upwards on the ball of the foot, while palpating the calf with his other hand. On the order to relax, softening of the calf combined with further dorsiflexion is evidence that dissociation is incomplete. By the time disociation is complete, favourable cases will have an active range of $20^{\circ}$ or more, and active dorsiflexion up to $80^{\circ}$ or $75^{\circ}$.

The phase of integration now begins, and partial weight bearing on crutches is introduced. For the first few days the backslab should be retained for wear at night, but after that it can be discarded. Dorsiflexion exercised with weights applied to the foot are advantageously continued throughout. The patient is taught to walk in a heel-to-toe manner, with exaggerated dorsi- 
flexion during 'swing through' of the operated foot. This is a time for vigilance on the part of the physiotherapist. Some patients 'forget' their carefully rehearsed dissociated dorsiflexion when faced with the complex pattern of walking, and integration of the new movement has to be deliberately and painstakingly practised. With time the pattern of walking becomes established, dissociation is maintained, and full weight bearing may then commence.

During the phase of weight bearing, the foot will 'drop' by about $5^{\circ}$, and any slight calcaneus deformity will disappear. The late dropping should be allowed for in the tensioning and immobilisation of the transfer at operation. A foot which appears to be too 'high' can be brought down by allowing partial weight bearing a little earlier than would otherwise be indicated and by discarding the back-slab early.

The patient is ready for discharge when, after some days of weight bearing, it is clear that dissociated dorsiflexion has become firmly absorbed into the walking cycle. The elderly should remain under observation for a longer period. It takes them longer to learn the new walking sequence, and there is a danger of relapse into a 'confused' gait if they are discharged too soon.

A warning should be given against adoption of a rigid post operative timetable. It is true that favourable cases can be ready for partial weight bearing within seven days of plaster removal, and can be ready for discharge in three or four weeks, but rigid application of such a schedule will ruin many results. A fault of some therapists is to attempt to force cases through the post operative course in accordance with a preconceived schedule. Experience has shown that patients, who vary in age, intelligence, powers of concentration, and motivation towards rehabilitation very also in their rate of progress after surgery, and no two cases are exactly alike. The technique selected for the operation also has its effect. The time for advancement to the next stage of treatment must be decided in accordance with the criteria outlined above. If, at any time errors in reeducation are detected there should be no hesitation in starting treatment again from the beginning. It is better for post operative physiotherapy to occupy three months than for a patient to be hurried into the next stage before he is ready for it. Many of the late failures (i.e. patients presenting with high stepping gait and 'relapsed' drop at follow up) are attributable to hurried re-education and premature discharge from treatment.

Electromyographic studies on tibialis posterior after transfer have shown that at first the muscle discharges during both dorsiflexion and plantar flexion. As re-education proceeds, the discharge during plantar flexion diminishes, while electrical activity during dorsiflexion increases. Even when complete clinical dissociation is seen, slight electrical activity may persist during plantar flexion. This serves to remind that this muscle may always retain a 'memory' of its previous function, and that for some months after operation it retains a tendency to revert to its original pro-gravity function. For this reason it is wise to encourage the patient to practice

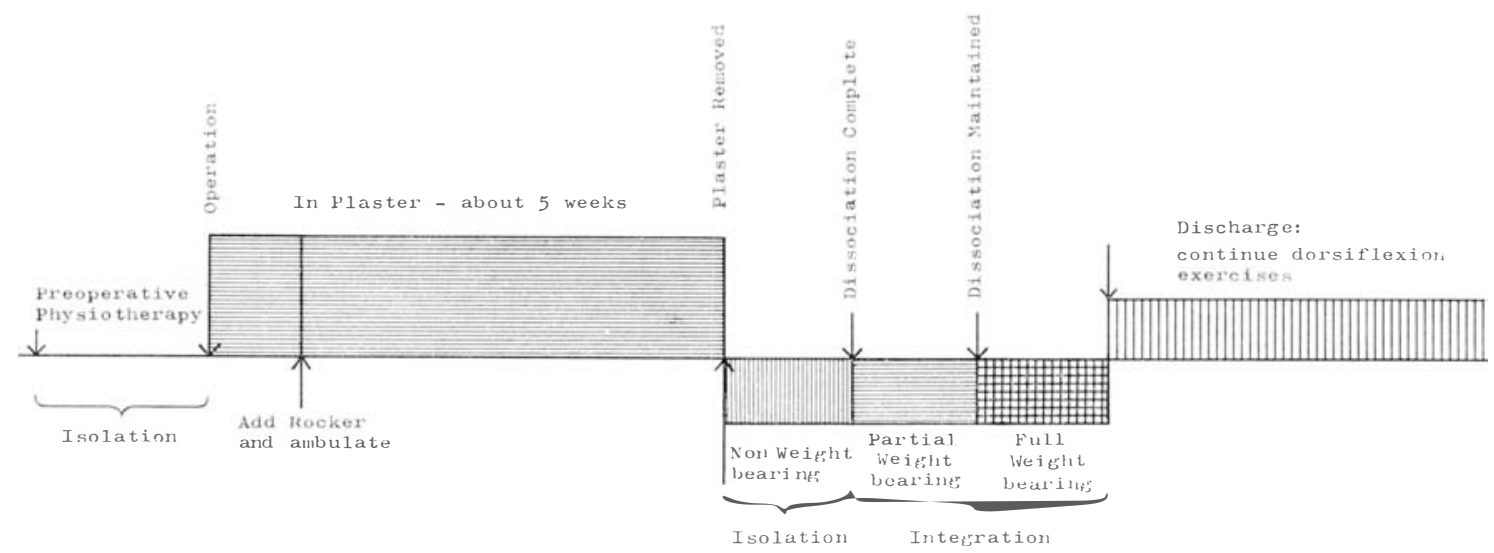


isolated dorsiflexion exercises against gravity with the foot weighted for at least six months after discharge, and to examine his gait, dissociation, and range of movements regularly for at least a year. In this way the incidence of late relapses may be minimised.

\section{S U M M A R Y}

The cardinal points in the physiotherapy of foot drop correction may be summarised by the following 'do's and 'don'ts'.

(I) Don't operate until the patient has achieved perfect isolation of the muscle.

(2) Don't start weight bearing until disociation is perfect.

(3) Do look our for (i) trick novements which may have deceived the therapist: (ii) failure to integrate the new movement when weight bearing is started.

(4) Don't hesitate to delay introduction of the next phase, or to order re-education again from the beginning if the patient exhibits 'confusion'.
(5) Don't apply a timetable to the patients' progress.

(6) Do see each patient regularly for at least a year from operation, and urge him to persist with dorsiflexion exercises.

\section{REFERENCES}

andersen, J . (1963). Acta. Ortho. Scand. 33 Fasc. 2 , i 5 I Andersen, J . (1964). Leprosy Rev., 35, 4I.

GunN, D. R. and molesworth, B. D. (1957). The Use of Tibialis Posterior as a Dorsiflexor. Journal of Bone and Joint Surgery 39-B, 674, 1957.

SElVAPANDiAn, A. J. and BRAND, P.w. (1959). Transfer of the Tibialis Posterior in Foot Drop Deformities. The Indian Journal of Surgery, 21. I 5 I, 1959.

WATKINS, M. B., JONES, J. B., RYDER, C. T., Jr., BROWN, T. B., Jr. Transplantation of the Posterior Tibial Tendon. Fournal of Bone and Joint Surgery, 36-A, I I 8 I. 1954

Lennox, w. M. (1964). The Surgical \& Physiotherapeutic Management of Foot Drop. Proc. Orthobaedic Section, Assoc. of Surgeons of India. December 1964.

mendes, m., Further Reading on Physiotherapy; Physiotherapy in Leprosy. John Wright \& Son, Bristol I 964 . 